\title{
The Dark Matter Content of Barred Spiral Galaxies
}

\author{
Glen Petitpas, Mousumi Das, Peter Teuben, \& Stuart Vogel \\ University of Maryland, Dept. of Astronomy, College Park MD, 20742, \\ $U S A$
}

\begin{abstract}
Two-dimensional velocity fields have been used to determine the dark matter properties of a sample of barred galaxies taken from the BIMA Survey of Nearby Galaxies (SONG). Preliminary results indicate that the maximal disk model is not appropriate in several galaxies in our sample, but higher resolution results will be needed to confirm this.
\end{abstract}

\section{Introduction}

Weiner et al. (2001a, 2001b) present a powerful technique for determining the mass of the bar (hence, the mass of the disk in strongly barred galaxies) by comparing perturbations in the gas velocity fields with barred galaxy simulations. Following the work of Weiner et al. (2001a, 2001b) we have undertaken a study using gas kinematics to determine the dark matter properties for a sample of barred galaxies observed with the BIMA SONG survey. Typically, our velocity fields have much higher spatial coverage than the $\mathrm{H} \alpha$ used by Weiner et al.

\section{Method}

K-band images from the 2MASS survey are reduced and cleared of point sources, deprojected, then symmetrized by averaging flipped images in the $\mathrm{x}$ and $\mathrm{y}$ axes. These images are used to compute the $2 \mathrm{D}$ gravitational potential using a FFT and an assumed constant scale height (see Figure 1a). The model is parametrized by assuming a mass-to-light ratio $(\gamma)$ and a softened isothermal halo to represent the complete potential in which the gas flow is traced. Large grids of the hydro simulations are run for a large number of free parameters. The hydro code is describe in detail in Piner, Stone, \& Teuben 1995. The simulations are run for 1.5 Gyr, at which point the gas flows have reached steady state. The models are then smoothed to match the resolution of the galaxy to which we are comparing (Figure 1b). A difference map is created between each of the models and the data, and a $\chi^{2}$ is computed and the best model is determined (see Figure 2).

\section{Results}

We have determined the best fit parameters for the galaxies NGC 3627 and NGC 5457. Based on our preliminary, low resolution simulations we find that the maximum disk approximation is not appropriate. If fact, for NGC 5457 we find that the minimum disk approximation fits the data best. For NGC 3627 


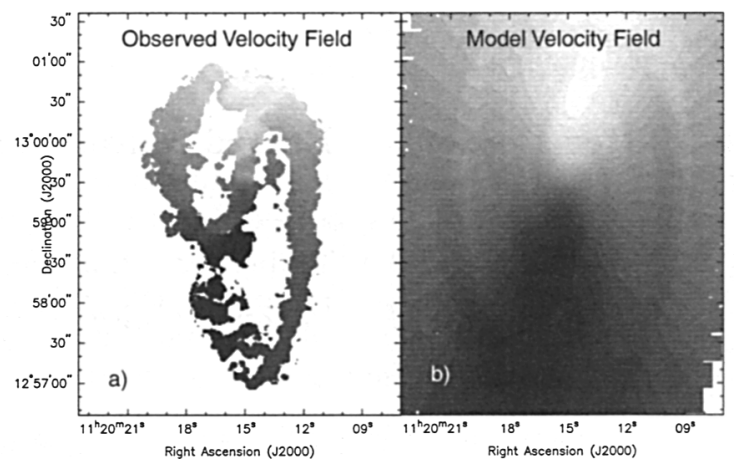

Figure 1. a) Observed CO velocity field of NGC 3627 taken from the BIMA SONG survey. b) The model velocity field derived from the 2MASS image and assumed dark matter halo.

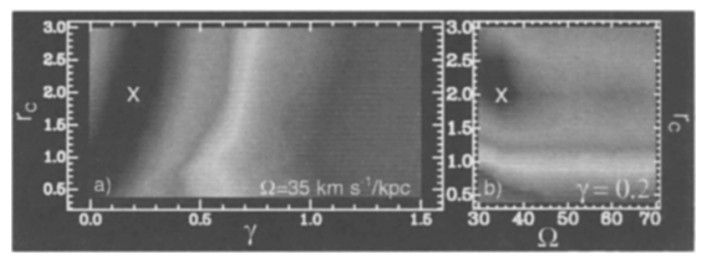

Figure 2. Slices of the $\chi^{2}$ cube through the best solution for NGC 3627. The darker shade indicates a better fit. The best solution is marked with an " $x$ ". a) Mass to light ratio plotted against dark matter core radius for the best bar pattern speed. b) Bar pattern speed plotted against dark matter core radius for the best value of mass to light ratio. We see little dependence on the bar pattern speed on the best-fit solution.

we find a mass-to-light ratio $\gamma=0.2$, a bar pattern speed $\Omega=35 \mathrm{~km} \mathrm{~s}^{-1} / \mathrm{kpc}$, and a dark matter core radius $r_{\mathrm{c}}=2.0 \mathrm{kpc}$ (see Figure 2).

We note here that these results are for the low resolution models and observations $\left(\sim 8^{\prime \prime}\right)$ only. We have obtained higher resolution data for all galaxies we intend to study and are currently generating high resolution similations for a large grid of galaxy parameters. Comparison of the high resolution observed $\mathrm{CO}$ velocity fields with these high resolution galaxy simulations will help clarify the results discussed here.

Acknowledgments. This work was supported by NSF grant AST 99-81289 and by the state of Maryland via support of the Laboratory for Millimeter-wave Astronomy.

\section{References}

Piner, B. G., Stone, J. M, \& Teuben, P. J, 1995, ApJ, 448, 508

Weiner, B. J., Williams, T. B., van Gorkum, J. H., \& Sellwood, J. A., 2001a, ApJ, 546, 916

Weiner, B. J., Sellwood, J. A., \& Williams, T. B., 2001b, ApJ, 546, 931 